\title{
INFRARED VARIATIONS OF ACTIVE GALAXIES: WHAT THEY TELL US
}

\author{
I.S. GLASS \\ South African Astronomical Observatory \\ PO Box 9, Observatory 7935, South Africa
}

Little is known about the long-term infrared variations of Seyfert galaxies. Are they large or small, fast or slow, regular or irregular? Do they possess variable components hidden at visible wavelengths? Can their variational time-scales give us information about activity on sub-milliarcsecond spatial scales? Do the infrared measurements show well-defined flux variation gradients? Is the infrared flux delayed with respect to changes in the output of the central engine? And do the infrared variability studies support the "Unified Model"?

The SAAO IR Monitoring Programme has usually included 36 galaxies and some have been monitored for up to 20 years. The sample comprises many Seyfert 1s, two Seyfert 2s and several S1 $<$ S $<$ S2 (x-ray galaxies). The wavelengths being monitored are $J(1.25 \mu \mathrm{m}), H(1.65 \mu \mathrm{m}), K(2.2 \mu \mathrm{m})$ and $L(3.5 \mu \mathrm{m})$. The aperture is kept constant at 12 arcsec diameter.

\section{Examples}

NGC 1068 - A hidden flux increase. NGC 1068 is the archetypal Seyfert 2 , believed until recently to be non-variable. Broad lines found in polarized light by Antonucci \& Miller (1985) imply it has a hidden Seyfert 1 nucleus. Its infrared flux at $L$ was found to have doubled over 18 years, showing that the nucleus is compact. (Glass $M N R A S, \mathbf{2 7 6}, \mathrm{L} 65,1995)$.

NGC 526A - A Seyfert 1.9 galaxy with strong $x$-ray emission. It has no known optical variation, but the SAAO observations have shown it to be dramatically variable in the infrared. The effect is small at $J(1.25 \mu \mathrm{m})$ but strong at $L(3.5 \mu \mathrm{m})$. This is explicable as the result of extinction within NGC 526A and dilution of the shorter-wavelength infrared fluxes by the underlying galaxy. 
NGC 2992 This is a Seyfert 1.9. It is the X-ray source 2A 0943-140 and it has a superwind outflow - possibly the result of supernovae in a massive starburst. It experienced a unique outburst-like event in 1988 with total energy $\sim 6 \times 10^{43} \mathrm{~J}$. The maximum power output of the event was $\sim 1.66$ $\times 10^{36} \mathrm{~W}$. As the outburst faded, a gradual temperature decline was seen. A very tentative model is that of a supernova in a dense region such as has been advocated by Terlevich et al. (MNRAS, 255, 713, 1992). (See Glass, MNRAS, 1998, in press).

\section{Flux Variation Gradients}

Chołoniewski (Acta Astr, 31, 293, 1981) and later Winkler et al. (MNRAS, $\mathbf{2 4 7}, 659,1992$ ) found that the $U$ and $B$ fluxes of variable Seyfert galaxies are linearly related (when measured through a constant aperture). This allows the definition of a unique gradient, called the Flux Variation Gradient (FVG), for each galaxy and colour combination.

Glass (MNRAS, 256, 23p, 1992) determined FVGs in the near-infrared for the Seyfert 1 galaxy NGC 3783. Detailed results have also been obtained for NGC 1068, NGC 4593, NGC 2992 and NGC 7469. Data for all the galaxies in the SAAO programme have been reduced in a preliminary way to determine the distribution of FVG values.

The distribution of FVGs in each colour is narrow, indicating quasiuniversal colour temperatures around $1600 \mathrm{~K}$. The dispersion is least in $H$ $K$. Seyferts 1.9 and 2 tend to be redder than average, supporting the view that they are Seyfert $1 \mathrm{~s}$ viewed through intervening interstellar material.

The relative narrowness of the $H-K$ distribution is possibly because $K-L$ is affected by outer, cooler, dust while $J-H$ is contaminated by the tail of the UV component form the central engine. It is also the case, however, that the $J$ variation is small and the $L$ mags are more difficult to measure, so that observational errors may add to scatter.

\section{Delayed Response - Summary of Cases}

Clear case: Fairall 9 - 400 d (Clavel, Wamsteker, Glass, ApJ, 337, 236, 1989).

Fair cases: in NGC 3783 - 80d Mkn 744 - 37d (Nelson, ApJ, 465, L87, 1996); NGC 1566 - 2 mos (Baribaud et al. $A \& B A p$, 256, 375, 1992): GQ Com (QSO) - 700d (Sitko et al. ApJ, 409, 139, 1993).

Less certain cases: NGC 4151 - 18d (Oknyanskii, Astron Lett, 19, 416, 1993); III Zwicky 2 - 2yr (Lebofsky \& Rieke, Nature, 284, 410); MCG-258-22, Mkn509, 3A0557-383, ESO141-G55 (from hysteresis diagrams; Glass in IAU Symp 134, Active Galactic Nuclei, p382, 1989, Kluwer). 